\title{
X-ray fluorescence modelling for Solar system regoliths: Effects of viewing geometry, particle size, and surface roughness
}

\author{
Jyri Näränen ${ }^{1} \dagger$, Hannu Parviainen ${ }^{1}$ \\ and Karri Muinonen ${ }^{1}$ \\ ${ }^{1}$ Observatory, Tähtitorninmäki (PO Box 14), FI-00014 University of Helsinki, Finland
}

\begin{abstract}
Soft X-ray fluorescent emission from the surfaces of asteroids and other atmosphereless solar-system objects is studied using ray-tracing techniques. X-ray observations allow the assessment of the elemental composition and structure of the surface. The model regolith is assumed to consist of close-packed uniformly distributed spherical particles of equal size. The surface is also assumed to be rough according to a fractional-Brownian-motion model. The fluorescent X-ray emission from regolith surfaces is simulated in order to better understand the contribution of viewing-geometry -related phenomena on the signal obtained from, e.g., orbiting platforms. The first results are presented and the applicability of the methods to the interpretation of future asteroid and Mercury mission X-ray data (e.g., BepiColombo) is discussed.
\end{abstract}

Keywords. spectroscopy, Moon: asteroids, surface; X-rays, scattering

\section{Introduction}

Several atmosphereless solar-system bodies, including the Moon (Foing et al. 2006), and asteroids (433) Eros (Nittler et al. 2001) and (25143) Itokawa (Okada et al. 2006), have been studied in soft X-ray wavelengths, i.e., at energies $\sim 0.1-10 \mathrm{keV}$. The main objective since the first planetary X-ray observations with the Apollo program (Adler et al. 1972) has been to obtain elemental ratios (e.g., $\mathrm{Mg} / \mathrm{Si}, \mathrm{Al} / \mathrm{Si}$ ) and absolute abundances from fluorescent emission induced by solar irradiation. These measurements allow the determination of the elemental composition of the surface layer of the object which is crucial for establishing the mineralogy of the surface.

The past studies have been somewhat limited in spatial and spectral resolution because of the detector technology available. As a consequence, viewing-geometry-related phenomena have not been studied nearly as much as in, e.g., the visible wavelengths. With the advent of new CCD-based X-ray spectrometers (Grande et al. 2002) and microchannel optics suitable for space missions, the situation is changing. The future missions (especially to objects close to the Sun, e.g., NEOs and Mercury) will deliver much more detailed data than has been available thus far and, as a result, more detailed analytical tools are needed. The ESA mission to Mercury, BepiColombo, for example, will carry an $\mathrm{X}$-ray spectrometer capable of sub-kilometer resolution at best.

In the visual wavelengths, the use of viewing-geometry-related phenomena for derivation of physical parameters, e.g., particle size and surface roughness, has been extensively studied. Some research has also been carried out to understand how viewing geometry affects the soft X-ray signal, but so far the data has not been good enough to give

$\dagger$ email: naranen@astro.helsinki.fi 
conclusive results. The study by Okada \& Kuwada (1997) shows that the roughness and size distribution of the surface regolith affect the signal at large phase angles. Okada (2004) has also shown that the NEAR-Shoemaker X-ray analysis on the mineralogy of asteroid 433 Eros can be improved if the phase angle of the observations is taken into account. The characteristic elemental fluorescent lines are affected at different rates, making the relative abundance studies more complex than previously considered.

Much of the background in planetary X-ray data is caused by different forms of viewinggeometry-dependent scattering (both elastic and inelastic). In this paper, we will not concentrate on scattering, but it will be an important addition to our studies in the near future.

In the present paper, we describe first results from our studies of particle size, surface roughness, and viewing-geometry effects on soft X-ray fluorescence from a realistic medium. We employ a ray-tracing method to simulate the fluorescence from a semiinfinite plane-parallel medium consisting of spheres of equal size and fixed volume fraction. Surface roughness is approximated by weighting the ray-tracing results with a shadowing function produced with a fractional-Brownian-motion surface model.

First, we outline the methods employed in our studies. We then proceed to presenting the first results. We conclude by discussing the importance of this study to future space missions and by outlining the plans for future work.

\section{Theoretical and numerical models}

We employed two different approaches in this study to replicate the fluorescent signal expected from a regolith surface of an atmosphereless object. First, the signal itself was produced by using ray-tracing simulations on a surface model consisting of spheres of equal size with known volume fraction (ratio of the volumes of the particles and to the full medium). Second, we took into account the more macroscopic effect of surface roughness. This we achieved by producing shadowing functions from a fractional Brownian-motionsurface model.

Here we concentrate on fluorescent emission and omit, e.g., Bragg scattering. Also secondary fluorescence, i.e., fluorescence induced by fluorescent radiation of higher energy, is not included.

\subsection{Ray-tracing fluorescence simulations}

Fluorescent emission was simulated by tracing rays through a medium made of spherical particles of equal size. The method is similar to that by Muinonen et al. (2001) with the largest differences in the interaction of rays with the particles. In the study at hand, the free path of the ray in the medium was Monte Carlo sampled and whenever the ray encountered a particle the occurrence of absorption was studied. In the case of absorption, a fluorescent yield factor was applied. If fluorescence happened the ray was emitted isotropically and traced until it was absorbed again or it escaped from the medium, in which case the emitted direction and flux density was registered (Muinonen et al. 2004).

The volume fraction of the medium was kept at 0.3 in all of the simulations. As a light source we used simulated solar spectrum during X-flare (cut-off at $30 \mathrm{keV}$ ) (Juhani Huovelin, private communication). The light source was assumed to be in nadir. This allowed the averaging of the results over all azimuth angles.

The number of particles per surface elements was adjusted according to the size of individual particles to stop rays from going through the medium. The average number of particles was 2000-10000 per surface element. To prevent artificial features from structures in the simulated medium, one hundred random media with fixed volume fraction 
were created for each simulation, i.e. each of these media were targets for $1 \%$ of the total number of rays used. To get good statistics, several million rays are required. This requirement was fulfilled for all the other particle sizes except $1 \mu \mathrm{m}$ for which we had to contend with 750000 rays due to computational limitations.

As for the fluorescent material, we concentrated on two elements that have been found in planetary soft X-ray spectra, iron ( $\mathrm{Fe}$ ) and calcium (Ca). However, other materials can also be easily implemented.

We validated the simulation algorithm by comparing it with radiative transfer results for isotropic scatterers (a modification of the work by Muinonen 2004).

\subsection{Rough surface modelling}

Surface roughness can have a significant effect on the observed radiation interacting with the surface. The surface features can shadow the surface from incident radiation, and mask the escaping radiation from the irradiated parts. For a homogeneous isotropic surface with certain roughness statistics, the geometric self-shadowing and self-masking can be combined into a shadowing function $S\left(\theta_{i}, \phi_{i}, \theta_{e}, \phi_{e}\right)$. The shadowing function gives the probability for a point visible to the observer to be illuminated as a function of the incident angles $\left(\theta_{i}, \phi_{i}\right)$ and the emergent angles $\left(\theta_{e}, \phi_{e}\right)$ (Shepard \& Campbell 1998).

Exact formulation of the combined shadowing and masking function is currently possible only for a few simplified surface-roughness models, but true natural rough surfaces show complex roughness features in all scales of examination. This sort of self-affine fractal behaviour cannot currently be handled with analytical methods, and models based on numerical simulations are required.

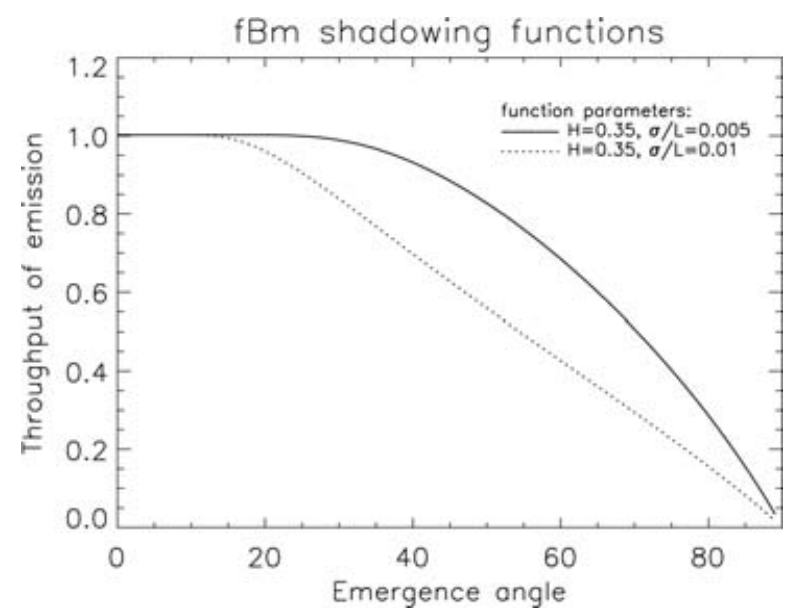

Figure 1. Shadowing functions used in our study. The emergence angle is the angle measured from the surface normal in which the ray emerges from the medium.

For creating shadowing functions for this study we employed a fractional-Brownianmotion surface model by Parviainen \& Muinonen (2006); see also Peitgen \& Saupe (1988). The parameters used in the model represent the standard deviation of the surface heights normalized to element width $(\sigma / L)$ and Hurst exponent $(H)$ which determines the selfaffine fractal roughness features of all the length scales. 

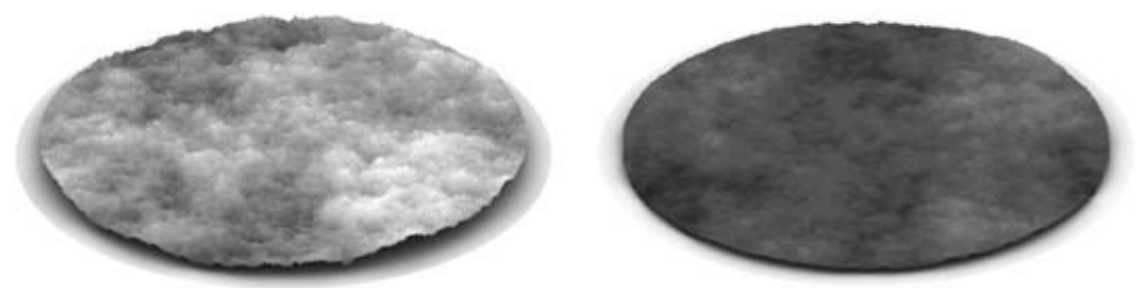

Figure 2. An illustration of the rough-surface models. The Hurst exponent $H$ and height standard diviation $\sigma / L$ are as follows: $H=0.35, \sigma / L=0.01$ (left); $H=0.35, \sigma / L=0.005$ (right).

\section{Results}

\subsection{Effect of grain size}

For the study of the effect of grain size on the fluorescent emission, we report results produced with iron ( $\mathrm{Fe}$ ) particles, but similar effects were also seen for calcium (Ca). We noted overall increase in the fluorescent radiation with decreasing particle size. The increase is most notable in particle sizes of $10 \mu \mathrm{m}$ and less. For example, the fluorescent emission at $60^{\circ}$ emergence angle is $\sim 2.6$ times greater for $1 \mu \mathrm{m}$ particles than for 250 $\mu \mathrm{m}$ particles.

A nonlinear brightening near the opposition geometry was also observed. We will address this opposition effect later on. There is also a notable nonlinear brightening in larger emergence angles. This increase is also observed in the case of isotropic scattering in the visible wavelengths. It will, however, cancel out once rough surfaces are introduced into the simulation as can be seen below.

The apparent scatter in the emission curves in Figure 3 is due to computational noise and not a real effect.

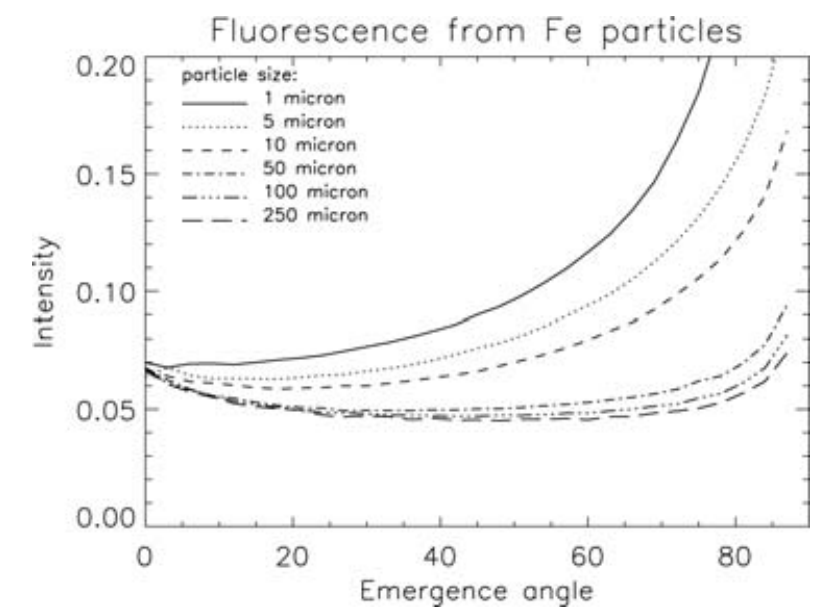

Figure 3. Fluorescent emission from iron-particle media of different particle sizes.

\subsection{Variation of apparent elemental ratios with changing viewing geometry}

The NEAR-Shoemaker X-ray spectrometer (XRS) obtained data of asteroid 433 Eros in a large variety of different viewing conditions (Trombka et al. 2000). The initial analysis did not, however, produce conclusive results for mineralogical composition. This can be due to various reasons, but Okada (2004) has shown that the analysis can be improved if 
the viewing-geometry-dependent effects of particle size and mutual shadowing of particles (micro-scale surface roughness) are taken into account.

In our study, we assumed two media of the same dimensional properties but different elemental composition. The first consisted of iron and the second of calcium. We then produced the $\mathrm{Fe} / \mathrm{Ca}$ emission ratio as a function of viewing geometry for the case of equally sized particles and the case where the iron particles were 10 times larger. No shadowing functions were included in this part of the study, since macro-scale shadowing is independent of particle sizes and elemental compositions.

In both, cases a change in the elemental ratio as a function of viewing geometry was seen. This can be understood by the different mean free paths of radiation for the different elements in the media, i.e., iron absorbs photons more efficiently thus making it easier for fluorescent photons to escape in small emergence angles in comparison to larger angles. In the case of unequal particle sizes, an enhancement of the effect was observed due to the particle-size effect on fluorescence. The two cases presented here illustrate well the complexity of the analysis of elemental ratios from X-ray spectrometer data.

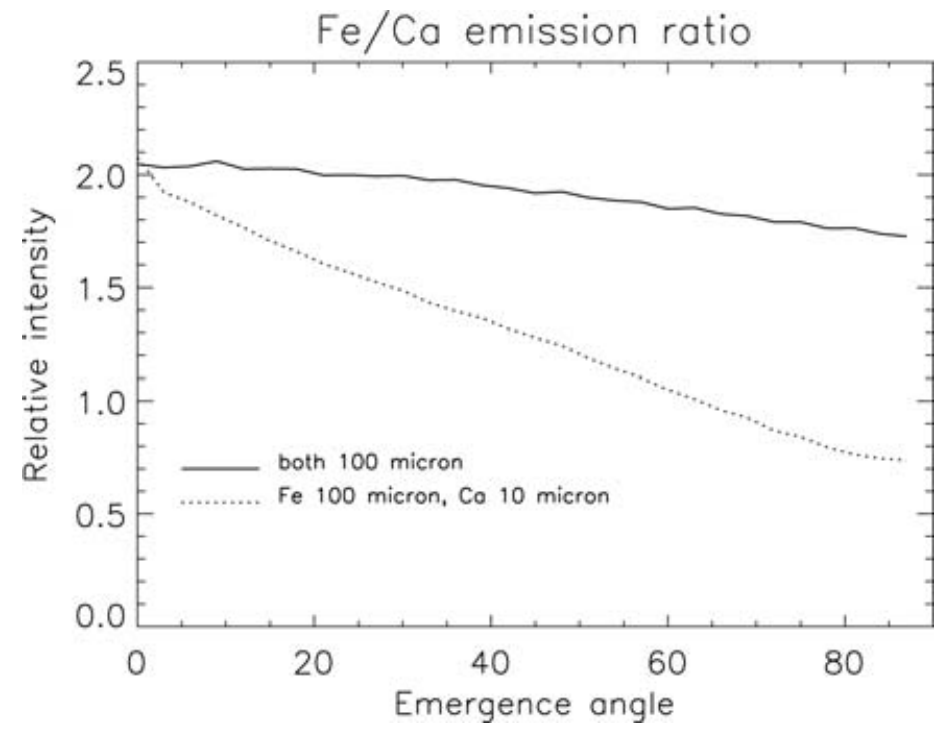

Figure 4. The Fe/Ca ratio of emitted fluorescent radiation as a function of viewing geometry. The two cases are: 1) both elements have equal particle size; 2) Ca particles are smaller by an order of magnitude.

\subsection{Opposition effect}

When the viewing geometry is close to the opposition geometry, i.e., the observer and the illumination source are in the same direction, a nonlinear brightening was observed for all grain sizes. This is explained by the fact that, in opposition, the particles in the surface offer no obstruction for the escaping photons. The situation is analogous to the shadow-hiding opposition effect in visual wavelengths.

\subsection{Rough-surface shadowing}

The application of two shadowing functions obtained from the fractional-Brownianmotion surface model clearly smoothens out the brightening in the larger viewing angles. The effect can be almost an order of magnitude in the larger emergence angles (ratio of the intensities at $80^{\circ}$, when shadowing is and is not accounted for is $\sim 7$ ). 


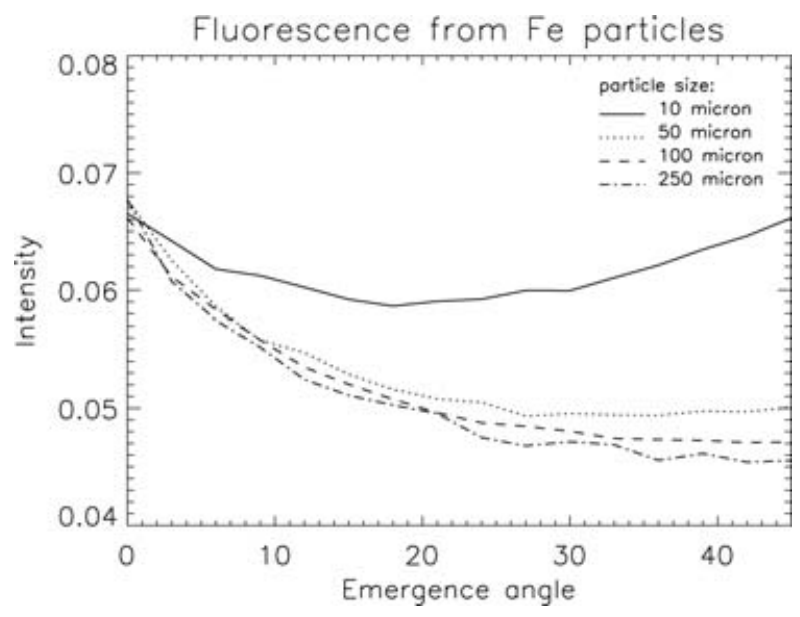

Figure 5. Brightening towards the opposition geometry.

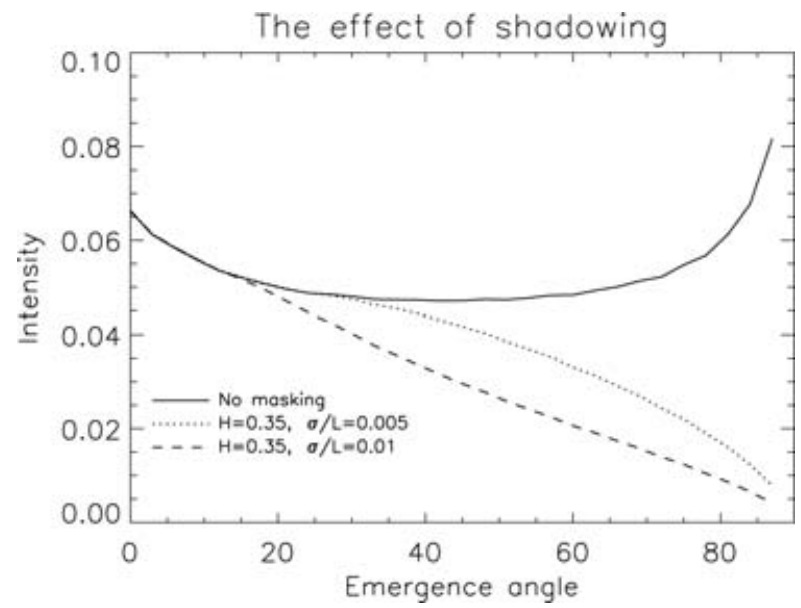

Figure 6. The effect of shadowing on the fluorescent emission of $100 \mu \mathrm{m}$ Fe particles. The parameters for the two shadowing functions are given in the figure.

\section{Conclusions and future work}

We have presented the first results from our study of viewing-geometry, particle-size and surface-roughness effects in soft X-ray wavelengths. The results show that the physics of soft X-ray fluorescence are complicated. Change in particle size can affect the amount of fluorescent emission in a given viewing geometry by as much as $100 \%$ or more. The ratio of fluorescent emission of two identical media made of different elements is a function of viewing geometry. It also depends on the sizes of the elemental particles.

This shows that the analysis of soft X-ray data obtained with spectrometers in the future with good spatial and spectral resolution requires more elaborate models than used until now. This is especially important for missions to asteroids, where the viewing geometry can change rapidly and cover a large number of viewing angles.

In addition to creating new problematics for detailed soft X-ray spectrometry, there is also potential for using viewing-angle-dependent phenomena as data for inversion of physical parameters, e.g., surface roughness and particle sizes, of the surface. 
One of the most important improvements to our model in the future will be the implementation of secondary fluorescence, i.e., in allowing the possibility of fluorescent photons to be absorbed and re-emitted at lower energy levels. This also allows us to consider particles consisting of several elements thus providing a more realistic fluorescing medium. Developing a medium of particles with different size distributions for the simulations is planned. The effect of volume fraction needs to be studied in more detail. The effect of different types of Solar fluxes will also be addressed. It will be also interesting to study the NEAR Shoemaker XRS data and verify that the results reported by Okada (2004) can be replicated with our model.

Laboratory measurements with a soft X-ray spectrometer capable of changing the viewing geometry would provide important ground reference to our studies and is under consideration.

Scattering, both elastic and inelastic, forms a major contribution in the measured background. Thus, it will be important in the future to also include scattering in our modeling. Several forms of scattering need to be investigated including, e.g., Bragg scattering and classical electromagnetic scattering (in the softest part of the spectrum).

\section{Acknowledgements}

This work has been funded by the Academy of Finland grant 210607. We thank George Fraser for insightful discussions on the subject. Juhani Huovelin helped with the solar simulation. We would also like to thank Hsiang-Kuang Chang for a helpful review.

\section{References}

Adler, I., Trombka, J., Gerard, J., Lowman, P., Schmadebeck, R., Blodget, H., Eller, E., Yin, L., \& Lamothe, R. 1972, Science 175, 436

Bielefeld, M. 1977, in: 8th. Lunar Science Conference, Proc. Volume 1. (A78-41551 18-91) (New York: Pergamon Press, Inc.), p. 1131

Foing, B.H., Racca, G.D., Marini, A., Evrard, E., Stagnaro, L., Almeida, M., Koschny, D., Frew, D., Zender, J., Heather, J., Grande, M., Huovelin, J., Keller, H.U., Nathues, A., Josset, J.L., Mälkki, A., Schmidt, W., Noci, G., Birkl, R., Iess, L., Sodnik, Z., \& McManamon, P. 2006. $\operatorname{AdSpR} 37,6$

Grande, M., Dunkin, S., Heather, D., Kellett, B., Perry, C. H., Browning, R., Waltham, N., Parker, D., Kent, B., Swinyard, B., Fereday, J., Howe, C., Huovelin, J., Muhli, P., Hakala, P.J., Vilhu, O., Thomas, N., Hughes, D., Allayne, H., Grady, M., Russell, S., Lundin, R., Barabash, S., Baker, D., Clark, P.E., Murray, C.D., Christou. A., Guest, J., Casanova, I., d'Uston, L.C., Maurice, S., Foing, B., \& Kato, M. 2002, AdSpR 30, 1901

Muinonen, K., Nousiainen, T., Fast, P., Lumme, K., \& Peltoniemi, J. 1996, JQSRT 55, 577

Muinonen, K., Stankevich, D., Shkuratov, Yu.G., Kaasalainen, S., \& Piironen, J. 2001, JQSRT 70,787

Muinonen, K. 2004, Waves in Random Media 14, 365

Muinonen, K., Huovelin, J., Alha, L., Lagerkvist, C.-L., Warell, J., Koskinen, H., Hämäläinen, K., Laukkanen, J., Nenonen, S., Andersson, H., Ritala, J., Stenberg, J., Korpela, S., \& Heilimo, J. 2004, in: Proceedings of the XXXVIII Annual Conference of the Finnish Physical Society (Department of Physical Sciences, University of Oulu, Finland, March 18-20, 2004), 4.18., abstract

Nittler, L., Starr, R., Lim, L., McCoy, T., Burbine, T., Reedy, R., Trombka, J., Gorenstein, P., Squyres, S., Boynton, W., McClanahan, T., Bhangoo, J., Clark, P., Murphy, M.E., \& Killen R. 2001, MaPS 36, 1673

Okada, T. \& Kuwada, Y. 1997, in: 28th. L\&PSC, abstract no. 1708

Okada, T. 2004, in: 35th. L\&PSC, abstract no. 1927

Okada, T., Shirai, K., Yamamoto, Y., Arai, T., Ogawa, K., Hosono, K., \& Kato, M. 2006, Science 312,1338

Parviainen, H. \& Muinonen, K. 2006, JQSRT, submitted 
Peitgen, H.O. \& Saupe, S. 1988, The Science of Fractal Images, Springer-Verlag

Shepard, M. \& Campbell, B. 1998, Icarus 134, 279

Trombka, J., Squyres, S., Brückner, J., Boynton, W., Reedy, R., McCoy, T., Gorenstein, P., Evans, L., Arnold, J., Starr, R., Nittler, L., Murphy, M., Mikheeva, I., McNutt Jr, R., McClanahan, T., McCartney, E., Goldsten, J., Gold, R., Floyd, S., Clark, P., Burbine, T., Bhangoo, J., Bailey, S., \& Petaev, M. 2000, Science 289, 2101 